\title{
Short-term effects of a nicotine-free e-cigarette compared to a traditional cigarette in smokers and non-smokers
}

Marco Ferrari ${ }^{1}$, Alessandro Zanasi ${ }^{2}$, Elena Nardi ${ }^{4}$, Antonio Maria Morselli Labate ${ }^{4}$, Piero Ceriana ${ }^{3}$, Antonella Balestrino ${ }^{3}$, Lara Pisani ${ }^{1}$, Nadia Corcione ${ }^{1}$ and Stefano Nava ${ }^{1,2^{*}}$

\begin{abstract}
Background: A few studies have assessed the short-term effects of low-dose nicotine e-cigarettes, while data about nicotine-free e-cigarettes (NF e-cigarettes) are scanty. Concerns have been expressed about the use of NF e-cigarettes, because of the high concentrations of propylene glycol and other compounds in the e-cigarette vapor.

Methods: This laboratory-based study was aimed to compare the effects of ad libitum use of a NF e-cigarette or and a traditional cigarette for 5 min in healthy adult smokers $(n=10)$ and non-smokers $(n=10)$.

The main outcome measures were pulmonary function tests, fraction of exhaled nitric oxide (FeNO) and fractional concentration of carbon monoxide (FeCO) in exhaled breath.

Results: The traditional cigarette induced statistically significant increases in FeCO in both smokers and non-smokers, while no significant changes were observed in FeNO. In non-smokers, the traditional cigarette induced a significant decrease from baseline in FEF75 ( $81 \% \pm 35 \%$ vs $70.2 \% \pm 28.2 \%, P=0.013)$, while in smokers significant decreases were observed in FEF25 (101.3\% $\% 16.4 \%$ vs $93.5 \% \pm 31.7 \%, P=0.037)$, FEV $1(102.2 \% \pm 9.5 \%$ vs $98.3 \% \pm 10 \%, P=0.037)$ and PEF (109.5 \% $\pm 14.6 \%$ vs $99.2 \% \pm 17.5 \%, P=0.009)$. In contrast, the only statistically significant effects induced by the NF e-cigarette in smokers were reductions in FEV $1(102.2 \% \pm 9.5 \%$ vs $99.5 \pm 7.6 \%, P=0.041)$ and FEF25 $(103.4 \% \pm 16.4 \%$ vs $94.2 \% \pm 16.2 \%, P=0.014)$.

Discussion: The present study demonstrated that the specific brand of NF e-cigarette utilized did not induce any majoracute effects. In contrast, several studies have shown that both traditional cigarettes and nicotinecontaining e-cigarettes have acute effects on lung function. Our study expands on previous observations on the effects of NF e-cigarettes, but also for the first time describes the changes induced by smoking one traditional cigarette in a group of never smokers.

Conclusions: The short-term use of the specific brand of NF e-cigarette assessed in this study had no immediate adverse effects on non-smokers and only small effects on FEV ${ }_{1}$ and FEF25 in smokers. The long-term health effects of NF e-cigarette use are unknown but worthy of further investigations.
\end{abstract}

Trial registration: Clinicaltrials.gov: NCT02102191

Keywords: Electronic cigarettes, Pulmonary function tests, Smoking, Concentration of carbon monoxide in exhaled breath

\footnotetext{
* Correspondence: stefanava@gmail.com

'Department of Specialistic, Diagnostic and Experimental Medicine,

Respiratory and Critical Care Unit, Alma Mater Studiorum, Sant'Orsola

Malpighi Hospital, University of Bologna, Bologna, Italy

${ }^{2}$ Respiratory and Critical Care Unit, Sant'Orsola Malpighi Hospital, Via

Massarenti 9, 40138 Bologna, Italy

Full list of author information is available at the end of the article
}

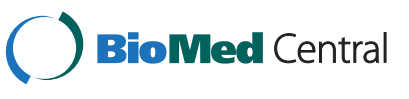

(c) 2015 Ferrari et al. Open Access This article is distributed under the terms of the Creative Commons Attribution 4.0 International License (http://creativecommons.org/licenses/by/4.0/), which permits unrestricted use, distribution, and reproduction in any medium, provided you give appropriate credit to the original author(s) and the source, provide a link to the Creative Commons license, and indicate if changes were made. The Creative Commons Public Domain Dedication waiver (http://creativecommons.org/publicdomain/zero/1.0/) applies to the data made available in this article, unless otherwise stated. 


\section{Background}

Electronic cigarettes (e-cigarettes) have been proposed as a novel method for quitting smoking. The producers of e-cigarettes claim that one of the benefits of these cigarettes is that a smoker may gradually decrease the nicotine content over time, until a state of "nicotine-free" smoking is reached. This state can be reached without the smoker having to renounce "habit-automatisms" and handling, which are major obstacles to quitting smoking.

However, the recent position statement of the Forum of International Respiratory Societies [1] on the use of e-cigarettes and their potential hazards concluded that these devices should be restricted or banned until more information about their safety is available. The major concerns include the nicotine content and the potential harm due to the high concentrations of propylene glycol, which is irritant when inhaled, chemicals, such as quinoline, benzoic acid and diethylcarbonate, and other compounds found in the e-cigarette vapor.

To our knowledge there are no data on the health effects of acute use of nicotine-free e-cigarettes (NF e-cigarettes); we, therefore, designed a study to compare the changes in pulmonary function tests (PFT) and fractions of exhaled nitric oxide (FeNO) and carbon monoxide (FeCO) as a result of 5 min of ad libitum smoking of a NF e-cigarette or a traditional cigarette, in smokers and non-smokers.

\section{Methods}

\section{Subjects}

Twenty normal subjects, recruited among pulmonary fellows or attending physicians were studied: 10 were smokers (minimum of 5 pack-years) and 10 were nonsmokers. Exclusion criteria were current use of any medication, the presence of any acute or chronic lung disease, neuromuscular diseases, cancer, chronic heart failure, metabolic or auto-immune diseases and acute illness during the preceding 4 weeks. Each subject was asked to sign written informed consent to the protocol approved by the Salvatore Maugeri Ethical Committee. The protocol was registered at www.clinicaltrials.gov with the number NCT02102191 on March 27, 2014.

\section{Protocol}

Both smokers and non-smokers were randomized to smoke both the NF e-cigarette and a commercial "popular brand" standard cigarette ad libitum for $5 \mathrm{~min}$ in two different sessions according to a cross-over design ( 5 patients within each group smoked first the NF e-cigarette and then the commercial cigarette and 5 subjects smoked first the commercial and then the NF e-cigarette). All subjects were asked to use a similar pattern and frequency of smoke aspiration, although it cannot be assured that they did so. The subjects were also asked to refrain from smoking in the $6 \mathrm{~h}$ preceding the test session and not to eat or drink for at least $4 \mathrm{~h}$ prior to the experimental procedure.

The first smoking session started 5 min after the baseline measurement of FeCO, FeNO and PFT. The second smoking session started after a wash-out of $24 \mathrm{~h}$ after the end of the first session. This wash-out period was to ensure that there was no carry-over effect. The measurements of FeNO, FeCO and PFTs were repeated immediately after each smoking session.

The NF e-cigarette used in this study, ELIPS C Series (Ovale Europe S.r.l., Desenzano del Garda, Brescia, Italy), was a brand commercially available in Italy. It was formed of a steel shell with a microprocessor powered by a battery, a filter and a removable cartridge. Among the six different types of cartridge available, we chose "Natur Smoke aroma Nocciola Antistress $0 \mathrm{mg} / \mathrm{mL}$ nicotina" (Angelica, Bologna, Italy), i.e., a nicotine-free liquid with a hazelnut flavorThe liquid of the cartridge is registered by the Italian Regulatory Agency and had the following composition: glycerin $>50 \%$, isotonic solution 5-10\%, magnesium chloride 1-5 \%, natural flavor $0.1-1 \%$, and vitamin B12 0.1-1\%. The specific kind of NF e-cigarette chosen in the current study followed an unbiased internet search for products available and produced in Italy (e.g.Dea, Flatech, Flavour Roma). Use of the Angelica liquid was finally decided mainly due to logistic convenience since it was produced in the same city (Bologna) of investigation.

The commercial standard cigarette, Marlboro Red Label Box (Philip Morris USA Inc., Miami, FL, USA), contained nicotine $0.8 \mathrm{mg}$, carbon oxide (CO) $10 \mathrm{mg}$ and tar $10 \mathrm{mg}$. According to the manufacturer [2], the components not exceeding $0.1 \%$ of the weight of the tobacco were acetic acid 0.01 , acetophenone 0.0001, ammonium hydroxide 0.3 , amyl butyrate 0.0001 , benzaldehyde 0.005 , benzoin 0.005 , benzyl alcohol 0.1, cellulose 9.3, calcium carbonate 4.6, monopotassium phosphate 1.4 , potassium citrate 0.3 , guar gum 0.1, and hercon70 0.1.

\section{Measurements}

The exhaled nitric oxide (FeNO) and fractional concentration of carbon monoxide in exhaled breath (FeCO) were measured using chemiluminescense analyzers (NIOX MINO, Aerocrine AB, Solna, Sweden and Micro Smokerlyzer, Bedfont Scientific Ltd., Rochester, Kent, Great Britain, respectively) with a computerized program. The FeNO analyzer was calibrated with certified NO mixtures (100 ppb) in nitrogen.

PFT were performed with a spirometer (Chestgraph HI-105 - CHEST M.I. Inc, Tokyo, Japan). The following parameters were recorded in the sitting position: forced vital capacity (FVC), forced expiratory volume in $1 \mathrm{~s}$ $\left(\mathrm{FEV}_{1}\right)$, forced expiratory flow (FEF) $25 \%, 50 \%$ and $75 \%$ and peak expiratory flow (PEF). Spirometry was 
performed following the recommendations of the American Thoracic Society/European Respiratory Society (ATS/ERS) guidelines [3].

For the FeNO recording, the subjects were studied in the sitting position wearing a nose-clip and were asked to inhale as deeply as they could, to total lung capacity, while breathing through a mouthpiece and then to exhale at a flow rate of about $50 \mathrm{~mL} / \mathrm{s}$, maintaining a constant mouth pressure of 4 to $5 \mathrm{~cm} \mathrm{H}_{2} \mathrm{O}$ for $10 \mathrm{~s}$, aided by visual feedback on the screen of the instrument.

Each of the three different measurements (FeNO, FeCO and PFT) were separated by intervals of about $45 \mathrm{~s}$.

\section{Statistical analysis}

Data are expressed as mean $\pm \mathrm{SD}$ or as frequencies. A Kolmogorov-Smirnov non-parametric test was applied to test the normality of the distributions. Two-way ANOVA was applied to the differences observed between basal values and those after smoking a traditional cigarette or an e-cigarette, considering smoking habit and the crossover design as factors. The effects estimated by ANOVA are reported together with their $95 \%$ confidence intervals (95\% CI). A two-tailed P value less than 0.05 is considered statistically significant. All analyses were performed using SPSS for Windows (ver. 21, IBM Corporation, Armonk, NY, USA).

\section{Sample size}

Prokhorov et al. found a decrease of $2.14 \%$ in the predicted value of $\mathrm{FEV}_{1}$ in 18 volunteer, regular smokers after smoking one traditional cigarette [4]. Since the standard deviation of the within-subject difference was not reported, we have estimated this value as $3.26 \%$ (one third of the value reported as the overall standard deviation in the study by Prokhorov et al.; i.e., $9.78 \%$ ) [4]. By comparing these values versus no effect of the e-cigarette, we had to study 20 subjects in order to be able to reject the null hypothesis with a probability (power) of 0.80 and a two-sided type I error probability of 0.05 . We, therefore, set the sample size as 20 subjects ( 10 smokers and 10 non-smokers), hypothesizing similar effects of smoking one traditional cigarette between smokers and non-smokers. The sample size was estimated by means of "PS Power and Sample Size Calculations" software (Version 3.0.43; Department of Statistics of the Vanderbilt University, Nashville, TN, USA; http://biostat.mc.vanderbilt.edu/twiki/bin/view/Main/PowerSampleSize) according to Dupont and Plummer [5].

\section{Results}

The subjects' characteristics are reported in Table 1.

All the subjects completed the study protocol. A few non-smokers reported mild adverse events such as dry cough $(n=3)$ and throat irritation $(n=2)$ when smoking traditional cigarettes.

\section{$\mathrm{FeCO}$ and $\mathrm{FeNO}$}

The $\mathrm{FeCO}$ values in the smokers and non-smokers are shown in Figure 1. As expected, baseline FeCO values were significantly higher in smokers than in nonsmokers $(\mathrm{P}<0.001$,two-way ANOVA). The signify

cance values, using the two-way ANOVA analysis, of the changes of $\mathrm{FeCO}$ values versus the baseline ones observed in smokers and non-smokers after smoking each type of cigarette, as well as the comparison between the traditional and e-cigarette, are also shown in Fig. 1 while the estimated effects of the two different types of cigarette in the overall population and the comparison of these effects between smokers and non-smokers, using the two-way ANOVA analysis, are presented in Table 2 . In the 20 subjects studied the traditional cigarette significantly increased $\mathrm{FeCO}$ values $(\mathrm{P}<0.001)$; this effect was significant in both groups of subjects (smokers $\mathrm{P}<0.001$; non-smokers $P=0.043)$. In contrast, the e-cigarette did not have any significant effects on $\mathrm{FeCO}$ (overall population $P=0.486$; smokers $P=0.226$; non-smokers $P=0.804$ ). The increase of $\mathrm{FeCO}$ values observed after smoking the traditional cigarette was significantly different from the effect of the e-cigarette (overall population $P<0.001$; smokers $P<0.001$; non-smokers $P=0.048$ ). As far as the comparison between smokers and nonsmokers is concerned, no significant differences were found (traditional cigarette $P=0.127$; e-cigarette $P=0.301$ ). Likewise, the difference observed between the two types of

Table 1 Subjects' characteristics

\begin{tabular}{|c|c|c|c|c|}
\hline & Overall & Smokers & Non-smokers & $P$ value \\
\hline Gender (M/F) & $11 / 9$ & $4 / 6$ & $7 / 3$ & $0.370^{\mathrm{a}}$ \\
\hline Age (mean $\pm S D$, years) & $39.3 \pm 12.6$ & $42.3 \pm 12.8$ & $36.2 \pm 12.3$ & $0.291^{\mathrm{b}}$ \\
\hline Weight (mean $\pm S D, k g)$ & $67.9 \pm 10.4$ & $63.5 \pm 10.1$ & $72.3 \pm 9.2$ & $0.056^{\mathrm{b}}$ \\
\hline Height (mean $\pm \mathrm{SD}, \mathrm{cm})$ & $169 . \pm 10.0$ & $163 \pm 7.4$ & $176 \pm 8.3$ & $0.002^{\mathrm{b}}$ \\
\hline BMI (mean $\left.\pm S D, k g / m^{2}\right)$ & $23.5 \pm 2.5$ & $23.7 \pm 3.1$ & $23.3 \pm 1.9$ & $0.704^{b}$ \\
\hline Smoke history (pack-years) - mean \pm SD (range) & - & $19.4 \pm 10.8(5-35)$ & - & - \\
\hline
\end{tabular}

${ }^{\mathrm{a}}$ Fisher's exact test

${ }^{\mathrm{b}} t$-test for equality of means 


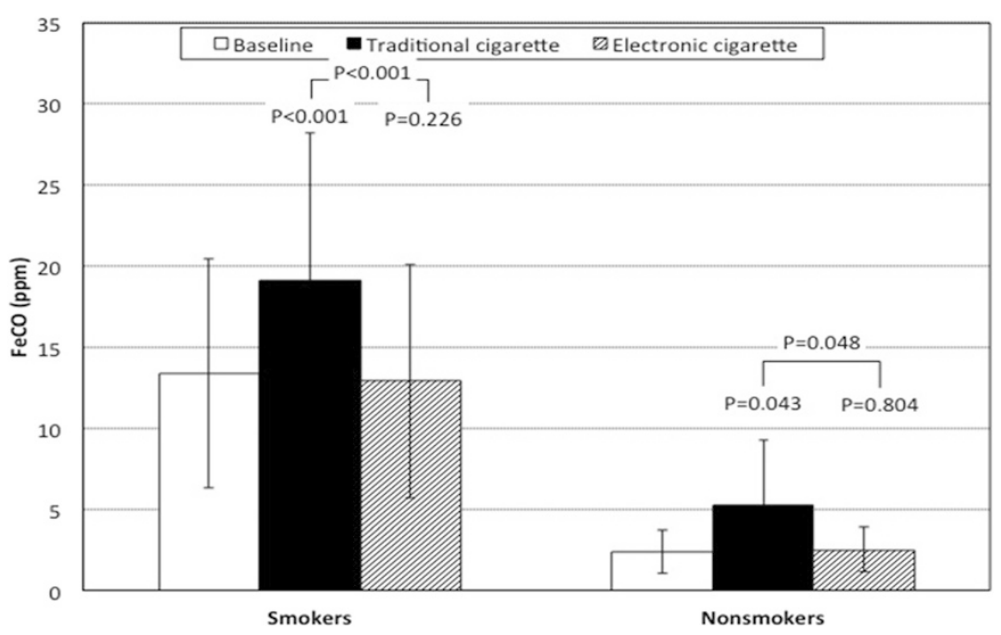

Fig. 1 Changes in fractional concentration of carbon monoxide in exhaled breath (FeCO) in smokers and non smokers

cigarette was not significant between smokers and nonsmokers $(P=0.067)$.

Baseline values of FeNO were not significantly different between smokers and non-smokers $(P=0.245$, two-way ANOVA). No significant changes of FeNO were observed in the two groups of subjects after smoking either a traditional or e-cigarette (Fig. 2, two-way ANOVA) and no significant changes were found in the overall group of subjects studied (Table 2).

\section{Pulmonary function tests}

All baseline pulmonary function tests $\left(\mathrm{FEV}_{1}, \mathrm{FVC}\right.$, $\mathrm{FEV}_{1} / \mathrm{FVC}$, and PEF) were similar between smokers and non-smokers ( $\mathrm{P} \geq 0.157$,Two-way ANOVA).

Smoking a traditional cigarette significantly decreased the $\mathrm{FEV}_{1} / \mathrm{FVC}$ in non-smokers $(P=0.047$ two way ANOVA; Fig. 3). In addition, both types of cigarettes significantly decreased $\mathrm{FEV}_{1}$ values in smokers (traditional $P=0.037$; electronic $P=0.041$, two-way ANOVA) while the decreases in non-smokers were not significant; thus FEV1 decreased significantly in the overall population $(P=0.013$, Two-way ANOVA) after smoking a traditional cigarette while the effect of the e-cigarette did not reach a statistically significant level $(P=0.070$, Two-way ANOVA). Finally, the traditional cigarette significantly decreased PEF values in the overall population $(P=0.017$, Two-way ANOVA) due to effect in the smokers $(P=0.009$,Two-way ANOVA). The changes in $\mathrm{FEV}_{1}, \mathrm{FVC}, \mathrm{FEV}_{1} / \mathrm{FVC}$, and PEF between the two types of cigarettes were not significantly different in either smokers or non-smokers (Fig. 3) or, indeed in the overall population (Table 2).

As far as FEF values are concerned, the traditional cigarette significantly decreased FEF25, FEF50 and FEF75 in the overall population $(P=0.030, P=0.033$, and $P=$
0.040, respectively, two-way ANOVA; Table 2), particularly due to the significant reductions of FEF 25 in smokers $(P=0.037)$ and FEF75 in non-smokers $(P=0.013)$ while the reduction of FEF50 did not reach the significant levels in either smokers $(P=0.213)$ or non-smokers $(P=0.063)$ (Fig. 4). The only significant effect of the e-cigarette was a reduction of FEF25 in smokers $(P=0.014$, two-way ANOVA). Comparing the effects of traditional and ecigarette smoking, only a significantly greater reduction of FEF50 was found after traditional cigarette smoking in non-smokers ( $P=0.036$, two-way ANOVA).

As far as concerns the comparison between smokers and non-smokers, higher values of FEF75 were found after smoking an e-cigarette than after smoking a traditional cigarette, whereas the inverse was the case in smokers $(P=0.037$, two-way ANOVA) (Table 2, Fig. 4c).

\section{Discussion}

We found that the specific brand of NF e-cigarettes used in this study was not associated with major acute physiological changes, causing only small, albeit statistically significant decreases in FEF25 and $\mathrm{FEV}_{1}$ in the group of smokers. In contrast, smoking a traditional cigarette induced immediate bronchoconstriction in non-smokers.

Tobacco cigarettes are one of the most important risk factors for disease worldwide and the primary goal of tobacco control is to reduce the mortality and morbidity associated with its use.

E-cigarettes have gained popularity in the last few years, mainly because of the advertisements of their producers, who claim that smoking tar-free cigarettes is associated with reduced risk for the health.

A recent systematic review assessing the efficacy of ecigarettes included six experimental studies and six cohort studies. The authors concluded that the use of e-cigarettes 
Table 2 Effects of the two different types of cigarette

\begin{tabular}{|c|c|c|c|c|c|c|}
\hline & \multicolumn{3}{|c|}{ Overall population } & \multicolumn{3}{|c|}{ Smokers vs. non smokers } \\
\hline & Effect & $95 \% \mathrm{Cl}$ & $P$ & Effect & $95 \% \mathrm{Cl}$ & $P$ \\
\hline \multicolumn{7}{|l|}{ FeCO (ppm) } \\
\hline Traditional & 4.3 & (2.3 to 6.2 ) & $<0.001$ & 2.9 & $(-0.9$ to 6.7$)$ & 0.127 \\
\hline Electronic & -0.2 & (-0.8 to 0.4$)$ & 0.486 & -0.6 & $(-1.8$ to 0.6$)$ & 0.301 \\
\hline$P$ value & $<0.001$ & & & 0.067 & & \\
\hline \multicolumn{7}{|l|}{ FeNO (ppb) } \\
\hline Traditional & -0.6 & $(-1.6$ to 0.4$)$ & 0.219 & 1.4 & $(-0.6$ to 3.4$)$ & 0.155 \\
\hline Electronic & -0.2 & $(-0.8$ to 0.4$)$ & 0.512 & 0.8 & $(-0.5$ to 2.1$)$ & 0.198 \\
\hline$P$ value & 0.372 & & & 0.501 & & \\
\hline \multicolumn{7}{|l|}{$\mathrm{FEV}_{1}(\%)$} \\
\hline Traditional & -3.4 & $(-5.9$ to -0.8$)$ & 0.013 & -1.0 & $(-6.1$ to 4.1$)$ & 0.691 \\
\hline Electronic & -1.7 & ( -4.8 to 2.9$)$ & 0.070 & -2.1 & $(-5.7$ to 1.6$)$ & 0.250 \\
\hline$P$ value & 0.098 & & & 0.575 & & \\
\hline \multicolumn{7}{|l|}{ FVC (\%) } \\
\hline Traditional & -1.6 & ( -5.7 to 2.6$)$ & 0.437 & -3.9 & $(-12.2$ to 4.4$)$ & 0.331 \\
\hline Electronic & -1.0 & $(-4.8$ to 2.9$)$ & 0.602 & -2.2 & (-9.8 to 5.4$)$ & 0.543 \\
\hline$P$ value & 0.584 & & & 0.448 & & \\
\hline \multicolumn{7}{|l|}{$\mathrm{FEV}_{1} / \mathrm{FVC}$} \\
\hline Traditional & -1.9 & $(-3.9$ to 0.1$)$ & 0.065 & 2.0 & $(-2.0$ to 6.1$)$ & 0.304 \\
\hline Electronic & -0.9 & $(-2.5$ to 0.6$)$ & 0.226 & -0.1 & (-9.8 to 3.0$)$ & 0.926 \\
\hline$P$ value & 0.182 & & & 0.132 & & \\
\hline \multicolumn{7}{|l|}{ PEF (\%) } \\
\hline Traditional & -6.5 & $(-11.7$ to -1.3$)$ & 0.017 & -7.5 & $(-17.8$ to 2.9$)$ & 0.148 \\
\hline Electronic & -3.8 & (-9.2 to 1.6$)$ & 0.155 & -6.0 & $(-16.8$ to 4.8$)$ & 0.254 \\
\hline$P$ value & 0.145 & & & 0.692 & & \\
\hline \multicolumn{7}{|l|}{ FEF25 (\%) } \\
\hline Traditional & -5.8 & $(-10.9$ to -0.6$)$ & 0.030 & .4 .1 & $(-14.1$ to 6.2$)$ & 0.412 \\
\hline Electronic & -3.7 & ( -7.6 to 0.3$)$ & 0.066 & -7.1 & $(-15.0$ to 0.7$)$ & 0.073 \\
\hline$P$ value & 0.252 & & & 0.414 & & \\
\hline \multicolumn{7}{|l|}{ FEF50 (\%) } \\
\hline Traditional & -5.0 & $(-9.5$ to -0.4$)$ & 0.033 & 2.1 & $(-6.9$ to 11.1$)$ & 0.629 \\
\hline Electronic & -2.9 & $(-6.8$ to 1.1$)$ & 0.143 & -2.9 & $(-10.8$ to 5.0$)$ & 0.447 \\
\hline$P$ value & 0.161 & & & 0.096 & & \\
\hline \multicolumn{7}{|l|}{ FEF75 (\%) } \\
\hline Traditional & -6.1 & $(-11.9$ to -0.3$)$ & 0.040 & 9.3 & (-2.3 to 20.9$)$ & 0.107 \\
\hline Electronic & -4.8 & $(-11.2$ to 1.6$)$ & 0.132 & 3.6 & $(-16.4$ to 9.3$)$ & 0.562 \\
\hline$P$ value & 0.657 & & & 0.037 & & \\
\hline
\end{tabular}

can reduce the number of cigarettes smoked and withdrawal symptoms, but that it was associated with some adverse events, such as mouth and throat irritation, nausea, headache and dry cough [6].

E-cigarettes have been shown to induce immediate adverse physiological effects after short-term use, similar to those observed with tobacco smoking [7]. The statement of the Forum of International Respiratory Societies [1] on the use of e-cigarettes and their potential hazards concluded that, considering the above-mentioned facts, the use of these devices should be restricted until more information on their safety becomes available.

More recently, NF e-cigarettes have been released on the market with the aim of minimizing the 


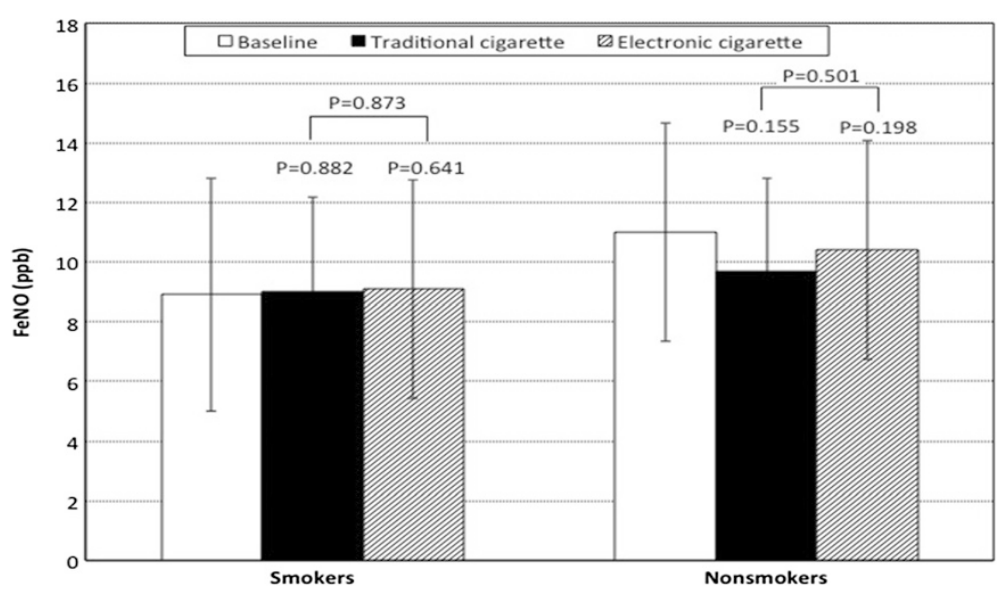

Fig. 2 Changes in fraction of exhaled nitric oxide (FeNO) in smokers and non smokers

adverse events linked with the use of nicotinedelivering e-cigarettes.

One of the concerns of using NF e-cigarettes is that the devices contain high concentrations of glycol, which is a known irritant when inhaled. Other potentially dangerous ingredients that may be found in NF e-cigarettes are solvents, genotoxins and various other chemicals and animal carcinogens (e.g., benzoic acid, quinoline).

The act of 'smoking' an e-cigarette is called 'vaping' and it mimics smoking so, in addition to delivering nicotine, it can address both pharmacological and behavioral components of cigarette addiction. Indeed, the

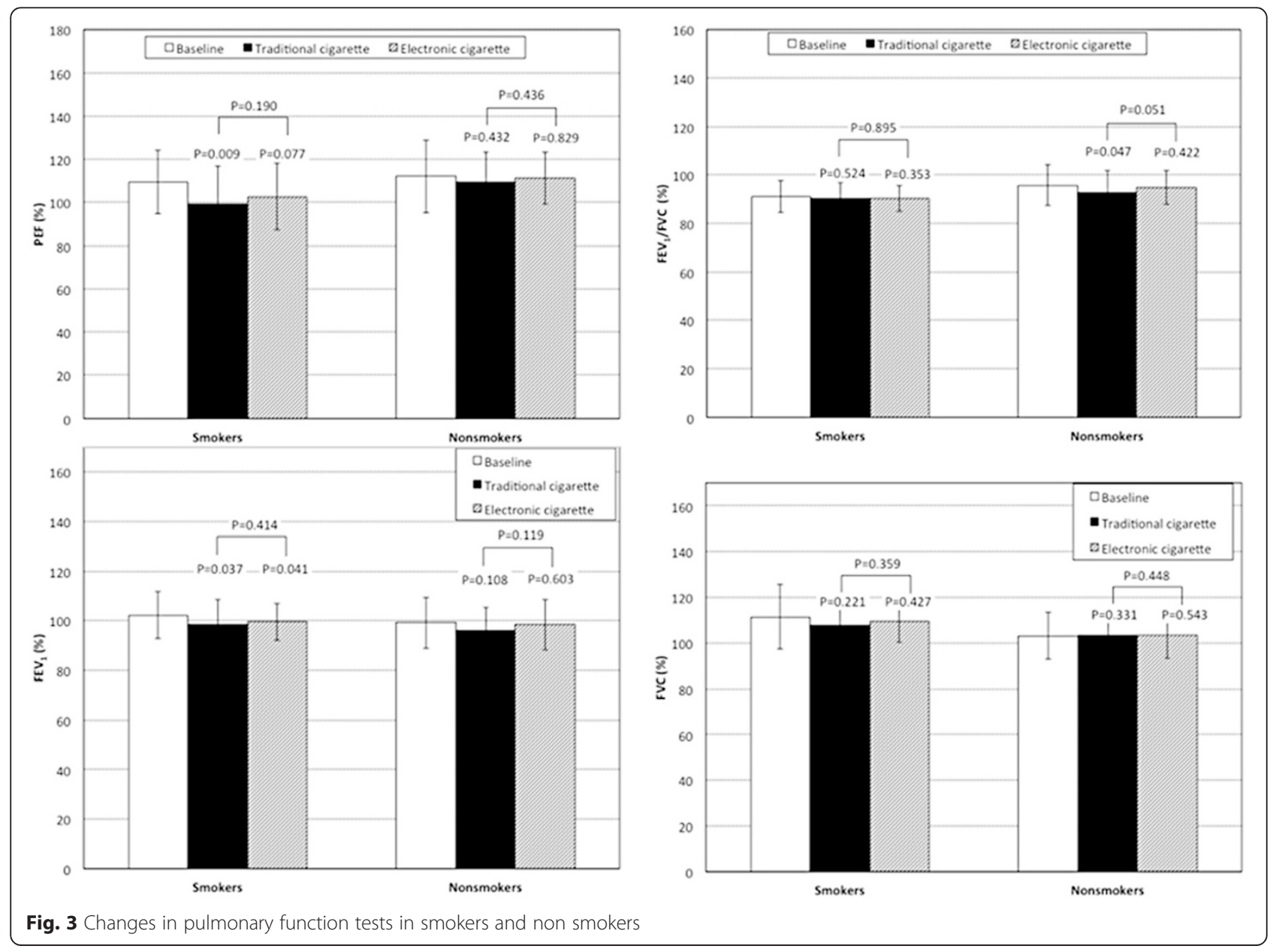




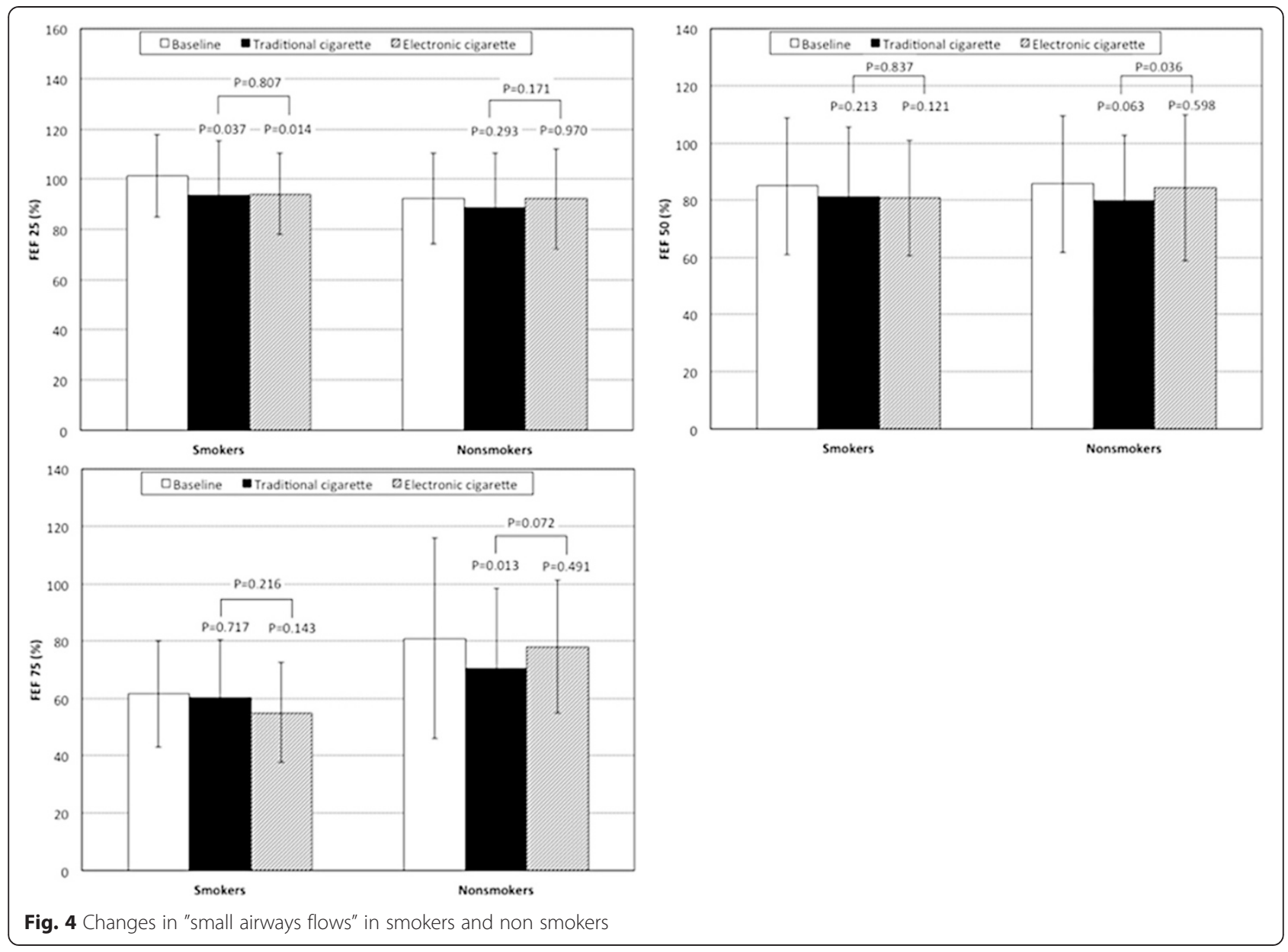

notable reduction in craving achieved by NF e-cigarettes demonstrates the ability of physical stimuli to suppress cravings independently of the administration of nicotine [8-13].

In a randomized, controlled trial of smoking cessation aided by nicotine e-cigarettes, NF e-cigarettes or nicotine patches performed in 657 people, Bullen et al. [13] found a rather disappointing percentage of abstinence at 6 months and the modest overall effect of the three methods (nicotine e-cigarettes, NF e-cigarettes, nicotine patches) did not allow a demonstration of the superiority of nicotine e-cigarettes over NF e-cigarettes.

The present study demonstrated that the specific brand of NF e-cigarette utilized did not induce any major acute effects. In contrast, several studies have shown that both traditional cigarettes and nicotinecontaining e-cigarettes have acute effects on lung function: Vardavas et al. [7] showed that e-cigarettes containing a dose of $11 \mathrm{mg}$ of nicotine significantly increased the impedance and resistance after $5 \mathrm{~min}$ of smoking, while Flouris et al. [12] demonstrated a significant immediate decrease in lung function only when smoking traditional cigarettes and not with e-cigarettes.
Similar data were also reported by Unverdorben et al. [14], who showed a significantly greater decrease in specific airway conductance and FEF25 after smoking a conventional cigarette than after a low nicotine $(5 \mathrm{mg})$ e-cigarette.

Our study expands on previous observations on the effects of NF e-cigarettes, but also for the first time describes the changes induced by smoking one traditional cigarette in a group of never smokers. Interestingly in these subjects the decreases in PFT values were much more pronounced (although not-significantly) than in the smokers, possibly because the airways of the former were more 'naïve' to noxious stimuli, which may have induced greater narrowing of the lumen of the peripheral airways, due to localized edema or smooth muscle contraction. The change in pulmonary function of the smokers was less pronounced than that reported in some previous studies and did not achieve statistical significance, despite the rate of change being quite similar to the above mentioned investigations. This may be due to the less sophisticated method of assessing airway narrowing in our study than in the study by Vardavas et al. (PTF vs impulse oscillometry, respectively) [7] or the 
longer and more intense habit of smoking of our group of subjects, which could have minimized the response.

The specific brand of NF e-cigarettes used in the present study did not induce any significant changes in smokers apart from decreases in $\mathrm{FEV}_{1}$ and FEF25. This latter finding is not easy to explain, and there are no investigations so far that have assessed the potential role of components of NF e-cigarettes on airway reactivity. Although both groups of subjects inhaled effectively - as illustrated by the significant increase in $\mathrm{FeCO}$ - only the group of active smokers reached levels of $\mathrm{CO}$ suggestive of deep inhalation (about $20 \mathrm{ppm}$ ), while the non-smokers had lower values (about $5 \mathrm{ppm}$ ). The higher levels of FeCO observed in smokers than in non-smokers may be explained by the previous $\mathrm{CO}$ exposure from tobacco smoking in the former. Indeed, according to the study protocol, smokers should have refrained from smoking in the $6 \mathrm{~h}$ preceding the experiments while the half-life of expired $\mathrm{CO}$ is about $4 \mathrm{~h}$, depending on exercise.

As expected, the FeNO level was reduced in the smokers, but no significant changes were observed in either group after cigarettes smoking. This is in contrast to what was observed in several studies [15-18], but not with the data of Chambers et al. [19] who found an increase in the level of exhaled NO minutes after smoking a traditional cigarette. Balint et al. [20] also found no significant change in the concentration of exhaled $\mathrm{NO}$ after smoking two cigarettes, but the concentrations of NO metabolites $\left(\mathrm{NO}_{2}^{-}+\mathrm{NO}_{3}^{-}\right)$were significantly increased. These findings suggest that NO might be trapped at the epithelial surface of airways in the formation of bioequivalent oxides of nitrogen such as peroxynitrite and S-nitrosothio.

The present study has some limitations that need to be discussed. First, the technique used to detect airway narrowing may not be as sensitive as the impulse oscillatory technique and it has been shown that changes in flow resistance usually precede variations in PFT. Indeed we assessed only crude spirometry, while DLCO and lung volumes and perhaps measurements of airway reactivity and particulate/vapor burden, may have given more insights into the problem. The ad libitum smoking of the cigarette may also be criticized because of the lack of standardization between subjects and because it is likely that a current smoker would smoke more than a non-smoker. The sample size (20 subjects) may be considered quite small, although it was based on a sophisticated calculation involving previously reported data $(4,5)$, so we are confident that the data collected may be representative enough of the changes induced by smoking either NF e-cigarettes or traditional ones.

\section{Conclusions}

In conclusion, smoking the NF e-cigarette studied in the present investigation had no immediate adverse effects after short-term use in non-smokers and a small effect on FEV 1 and FEF25 in smokers. In contrast, acute traditional cigarette smoking was associated with more detrimental effects on PFT in non-smokers than in smokers, although differences were not statistically significant. The long-term health effects of NF e-cigarette use are unknown but worthy of further investigation.

\section{Abbreviations \\ (NF e-cigarettes): Nicotine-free e-cigarettes; (FeNO): Fractional exhaled nitric oxide; (FeCO): Fractional concentration of carbon monoxide; (PTF): Pulmonary function tests.}

\section{Competing interests}

The authors declare that they have no competing interests.

\section{Authors' contributions}

$\mathrm{MF}=$ performed experiments, analyzed data and drafted the manuscript. He have given final approval of the version to be published. AZ = Gave substantial contributions to the conception and design of the study, data acquisition and performed experiments. He have given final approval of the version to be published. EN = data analysis and statistical analysis. Drafting of the manuscript. She have given final approval of the version to be published. $\mathrm{AMML}=$ statistical and data analysis. Gave substantial contributions to the conception and design of the study. He have given final approval of the version to be published. $P C=$ data analysis and drafting of the manuscript. He have given final approval of the version to be published. $A B=$ data analysis and drafting of the manuscript. She have given final approval of the version to be published. LP = performed experiments and collected data. Contributed to manuscript drafting. She have given final approval of the version to be published. NC = performed experiments and collected data. Contributing to the manuscript drafting. She have given final approval of the version to be published. SN = Principal Investigator. Designed the study, performed experiments, and wrote the manuscript. All authors read and approved the final manuscript.

\section{Author details}

${ }^{1}$ Department of Specialistic, Diagnostic and Experimental Medicine, Respiratory and Critical Care Unit, Alma Mater Studiorum, Sant'Orsola Malpighi Hospital, University of Bologna, Bologna, Italy. ${ }^{2}$ Respiratory and Critical Care Unit, Sant'Orsola Malpighi Hospital, Via Massarenti 9, 40138 Bologna, Italy. ${ }^{3}$ Respiratory Unit, Fondazione S.Maugeri, IRCCS, Istituto Scientifico di Pavia, Pavia, Italy. ${ }^{4}$ Laboratory of Biostatistics, Department of Medical and Surgical Sciences (DIMEC), Alma Mater Studiorum, University of Bologna, Bologna, Italy.

Received: 19 January 2015 Accepted: 21 September 2015 Published online: 12 October 2015

\section{References}

1. Schraufnagel DE, Blasi F, Drummond MB, Lam DC, Latif E, Rosen MJ, et al. Electronic Cigarettes: A position statement of the Forum of International Respiratory Societies. Am J Respir Crit Care Med. 2014;190(6):611-8.

2. http://www.philipmorrisusa.com/en/cms/Products/Cigarettes/Ingredients/ Tobacco_Flavor_Ingredients/default.aspx?src=top_nav

3. American Thoracic Society, European Respiratory Society. Standards for the diagnosis and management of patients with COPD. Available at: http:// www.thoracic.org/copd/pdf/copddoc.pdf. Accessed August 24, 2005

4. Prokhorov AV, Emmons KM, Pallonen UE, Tsoh JY. Respiratory response to cigarette smoking among adolescent smokers: a pilot study. Prev Med. 1996;25:633-40.

5. Dupont WD, Plummer WD. Power and Sample Size Calculations for Studies Involving Linear Regression. Control Clin Trials. 1998;19:589-601.

6. Gualano MR, Passi S, Bert F, La Torre G, Scaioli G, Siliquini R. Electronic cigarettes: assessing the efficacy and the adverse effects through a systematic review of published studies. J Public Health (Oxf). 2015;37(3):488-97.doi:10.1093/pubmed/fdu055. Epub 2014 Aug 9.

7. Vardavas Cl, Anagnostopoulos N, Kougias M, Evangelopoulou V, Connolly GN, Behrakis PK. Short-term pulmonary effects of using an electronic 
cigarette: impact on respiratory flow resistance, impedance, and exhaled nitric oxide. Chest. 2012;141(6):1400-6

8. Bullen C, McRobbie H, Thornley S, Glover M, Lin R, Laugesen M. Effect of an electronic nicotine delivery device (e cigarette) on desire to smoke and withdrawal, user preferences and nicotine delivery: Randomised cross-over trial. Tob Control. 2010;19(2):98-103.

9. Eissenberg T. Electronic nicotine delivery devices: Ineffective nicotine delivery and craving suppression after acute administration. Tob Control. 2010;19(1):87-8.

10. Barrett SP. The effects of nicotine, denicotinized tobacco, and nicotinecontaining tobacco on cigarette craving, withdrawal, and self-administration in male and female smokers. Behav Pharmacol. 2010;21(2):144-52.

11. Buchhalter AR, Acosta MC, Evans SE, Breland AB, Eissenberg, hhalter T. Tobacco abstinence symptom suppression: The role played by the smoking-related stimuli that are delivered by denicotinized cigarettes. Addiction. 2005;100(4):550-9.

12. Flouris AD, Chorti MS, Poulianiti KP, Jamurtas AZ, Kostikas K, Tzatzarakis MN, et al. Neither, Acute impact of active and passive electronic cigarette smoking on serum cotinine and lung function. Inhal Toxicol. 2013;25(2):91-101.

13. Bullen C, Howe C, Laugesen M, McRobbie H, Parag V, Williman J, et al. Electronic cigarettes for smoking cessation: a randomised controlled trial. Lancet. 2013;382(9905):1629-37.

14. Unverdorben M, Mostert A, Munjal S, van der Bijl A, Potgieter L, Venter C, et al. Acute effects of cigarette smoking on pulmonary function. Regul Toxicol Pharmacol. 2010;57(2-3):241-6.

15. Malinovschi A, Janson C, Holmkvist T, et al. Effect of smoking on exhaled nitric oxide and flow-independent nitric oxide exchange parameters. ERJ. 2006;28(2):339-45

16. Schilling J, Holzer P, Guggenbach M, Gyurech D, Marathia K, Geroulanos S. Reduced endogenous nitric oxide in the exhaled air of smokers and hypertensives. Eur Respir J. 1994;7:467-71.

17. Kharitonov SA, Robbins RA, Yates D, Keatings V, Barnes PJ. Acute and chronic effects of cigarette smoking on exhaled nitric oxide. Am J Respir Crit Care Med. 1995;152:609-12.

18. Marini S, Buonanno G, Stabile L, Ficco G. Short-term effects of electronic and tobacco cigarettes on exhaled nitric oxide. Toxicol Appl Pharmacol. 2014;278(1):9-15.

19. Chambers DC, Tunnicliffe WS, Ayres JG. Acute inhalation of cigarette smoke increases lower respiratory tract nitric oxide concentrations. Thorax. 1998:53:677-9.

20. Balint B, Donnelly LE, Hanazawa T, Kharitonov SA, Barnes PJ. Increased nitric oxide metabolites in exhaled breath condensate after exposure to tobacco smoke. Thorax. 2001;56:456-61.

\section{Submit your next manuscript to BioMed Central and take full advantage of:}

- Convenient online submission

- Thorough peer review

- No space constraints or color figure charges

- Immediate publication on acceptance

- Inclusion in PubMed, CAS, Scopus and Google Scholar

- Research which is freely available for redistribution 